\title{
Der Europäische Auswärtige Dienst - Chance oder Risiko für die europäische Entwicklungszusammenarbeit?
}

\author{
Von Julia Sattelberger, Heidelberg*
}

Die Europäische Union ist die weltweit finanzkräftigste Quelle von Entwicklungshilfegeldern. Ihre Bedeutung als globaler Akteur auf diesem Politikfeld wird jedoch durch eine komplizierte Struktur von Verantwortlichkeiten und Aufgaben zwischen den Organen der Union geschwächt. Kritik an der derzeitigen europäischen Entwicklungszusammenarbeit manifestiert sich im Vorwurf einer ineffizienten Organisation, die sich nachteilig auf die Umsetzung und Durchführung der europäischen Entwicklungszusammenarbeit auswirke. Der Vertrag von Lissabon schafft nun jedoch einen neuen primärrechtlichen Rahmen für dieses Politikfeld: Die Implementierung des Vertrages führt zur Restrukturierung und Neuschaffung von Institutionen, was nicht ohne Auswirkungen auf die künftige Steuerung und Durchführung der europäischen Entwicklungspolitik bleiben wird. Eine der institutionellen Neuerungen durch den Vertrag ist die Schaffung eines Europäischen Auswärtigen Dienstes (EAD).

Die genaue Ausgestaltung des neuen Dienstes lässt der Vertrag offen. Es heißt in Art. Artikel 27 Absatz 3 EUV lediglich, der EAD kooperiere mit den diplomatischen Diensten der Mitgliedsstaaten und setze sich zusammen aus Beamten der einschlägigen Abteilungen des Generalsekretariats des Rates und der Kommission sowie aus abgeordnetem Personal der nationalen diplomatischen Dienste. Die Organisation und die Arbeitsweise des Europäischen Auswärtigen Dienstes werde durch einen Beschluss des Rates festgelegt: "Der Rat beschließt auf Vorschlag des Hohen Vertreters nach Anhörung des Europäischen Parlaments und nach Zustimmung der Kommission." Entsprechend hat die derzeitige Hohe Vertreterin der EU für Außen- und Sicherheitspolitik, Catherine Ashton, am 25. März dieses Jahres einen Vorschlag zum Aufbau des neuen Europäischen Auswärtigen Dienstes vorgelegt. Bereits einen Tag später einigte sich der Ministerrat auf die künftige Organisation und Arbeitsweise des EADs, doch das Europäische Parlament verkündete direkt sein Missfallen an dem Vorschlag. Am 22. Juni konnte unter der Führung der spanischen Ratspräsidentschaft ein Kompromiss erzielt werden, der schließlich am 8. Juli vom Europäischen Parlament angenommen wurde.

Im folgenden soll Ashtons Vorschlag auf mögliche Konsequenzen für die Restrukturierung der institutionellen Architektur der Europäischen Entwicklungsverwaltung untersucht

Julia Sattelberger, M.A., Studium der Politikwissenschaf, wissenschaftliche Mitarbeiterin in der interdisziplinären Forschungsgruppe „Recht und Governance der Entwicklungszusammenarbeit“ am Max Planck Institut für ausländisches öffentliches Recht und Völkerrecht in Heidelberg. E-mail: jsattelb @mpil.de 
werden. Bedeutet die vorgesehene Umstrukturierung für die europäische Entwicklungspolitik eine Chance oder ein Risiko?

\section{Europäische Entwicklungspolitik vor Lissabon}

Die europäische Entwicklungszusammenarbeit war und ist Teil der außenpolitischen Aktivitäten der EU. Die Entwicklungszusammenarbeit unterstand vor der Implementierung des Vertrags von Lissabon vollständig der Verantwortung der Kommission. Entwicklungspolitische Kompetenzen waren hier zwischen dem Kommissar für Entwicklung und der Kommissarin für Außenbeziehungen und europäische Nachbarschaftspolitik aufgeteilt. Innerhalb der Kommission waren die Kompetenzen nach geographischer Zuständigkeit aufgeteilt.

Entsprechend war die Generaldirektion Entwicklung zuständig für Kooperationen mit den sogenannten AKP-Staaten aus Afrika, der Karibik und des Pazifik. Die Gelder für die Kooperationen mit den AKP-Staaten stammen aus dem Europäischen Entwicklungsfonds. Dieser wird nicht aus dem Budget der EU finanziert, sondern separat von den Mitgliedsstaaten gefüllt. Den rechtlichen Rahmen, auf dem die Kooperation der jetzigen EU mit den AKP-Staaten basiert, regelt das Cotonou-Abkommen - dem Nachfolger des LoméAbkommens. Dabei handelt es sich um einen Vertrag, der zwischen der Europäischen Gemeinschaft, deren Mitgliedsstaaten und den Ländern der AKP-Regionen multilateral ausgehandelt wurde. In der Entwicklungszusammenarbeit ist das die Ausnahme; in der Regel handelt es sich um einseitig vom Geber gesetztes Recht.

Die Kooperation mit Ländern in Asien und Lateinamerika, sowie Ländern, die in die Nachbarschaftspolitik der EU integriert waren, koordinierte dagegen die Generaldirektion Außenbeziehungen. Die Gelder für Kooperationen mit den Nicht-AKP-Staaten stammen aus dem EU-Budget. Wichtigstes Finanzierungsinstrument ist hier das Instrument für Entwicklungszusammenarbeit (DCI). Den rechtlichen Rahmen regelt hier die sogenannte DCIVerordnung, im Gegensatz zum Cotonou-Abkommen einseitig von der EG gesetztes Recht.

Geplant wird die Entwicklungszusammenarbeit in Form von Programmen in unterschiedlichen Planungs- bzw. Programmierungs-Schritten. Bis zum Vertrag von Lissabon wurde die Programmierung entsprechend der Länder- Zuständigkeit in den beiden Generaldirektionen durchgeführt. Entsprechend erfolgten alle Programmierungsschritte in der Kommission. Die Programmierungsverfahren für AKP- und Nicht-AKP-Staaten waren dabei ähnlich strukturiert, allerdings wurden die AKP-Staaten formal deutlich stärker in den Planungsprozess eingebunden als die Nicht-AKP Staaten. Durchgeführt wurde die Entwicklungszusammenarbeit schließlich gemeinsam mit der Durchführungsorganisation EuropeAid.

\section{Europäische Entwicklungspolitik nach Lissabon}

Der Vertrag von Lissabon macht eine strukturelle Reorganisation der europäischen Außenbeziehungen notwendig. Das betrifft namentlich das Amt der Hohen Vertreterin der Euro- 
päischen Union für Außen- und Sicherheitspolitik. Ihre Aufgabe wird es künftig sein, der Außendarstellung der EU ein einheitliches Gesicht zu geben und so die Kohärenz der europäischen Außenpolitik zu stärken.

Die Hohe Vertreterin ist zugleich Vizepräsidentin der Kommission, Vorsitzende des Rates für Auswärtige Angelegenheiten und Außenbeauftragte des Europäischen Rates. In ihren Zuständigkeitsbereich fallen die früheren Aufgaben des Kommissars für Außenbeziehungen und des Hohen Vertreters für die Außen- und Sicherheitspolitik. Entsprechend geht zumindest ein Teil des bisherigen Postens des Kommissars für Außenbeziehungen in dem Posten der Hohen Vertreterin auf. Langfristig ist zudem die Verschmelzung der Generaldirektion Außenbeziehungen in den künftigen Europäischen Auswärtigen Dienst vorgesehen. Die Bildung des neuen Dienstes erweist sich bisher als schwieriger und zäher Prozess. Letztlich geht es um die Verteilung von Kompetenzen und Einfluss. So wundert es kaum, dass die beteiligten Akteure versuchen, ihre Position möglichst erfolgreich in den Prozess einzubringen.

\section{Die mögliche entwicklungspolitische Architektur Europas - drei Perspektiven}

Insbesondere in Bezug auf die Frage nach der künftigen Aufteilung von Kompetenzen wird es aus entwicklungspolitischer Perspektive interessant: Konkret geht es hier vor allem um die künftige Kontrolle über finanzielle Instrumente aus den Entwicklungsbudgets. Vereinfachend lassen sich hier zwei grundsätzlich gegenläufige Positionen festhalten: Auf der einen Seite steht die Kommission, die unwillig ist, Verantwortung und Einfluss an den EAD abzutreten, da sie dadurch an Einfluss verlieren würde. Dagegen versuchen einige Mitgliedsstaaten, den EAD mit möglichst vielen Kompetenzen auszustatten. Vor diesem Hintergrund ergeben sich nun drei mögliche Szenarien zur Stellung der Entwicklungsverwaltung durch die Etablierung des EADs.

Eine Möglichkeit wäre der Aufbau einer starken entwicklungspolitischen Stimme innerhalb der Kommission. Im Hinblick auf die Verteilung von Kompetenzen zwischen der Kommission und dem EAD sieht dieser Vorschlag die Auflösung der regionalen Aufteilung entwicklungspolitischer Kompetenzen vor. Alle entwicklungsrelevanten Instrumente würden in diesem Sinne künftig einheitlich unter dem Dach der Generaldirektion Entwicklung zusammengefasst. Der Europäische Auswärtige Dienst stünde in einem solchen Szenario zwar in engem Kontakt zu den Implementierungsorganisationen, er hätte aber kaum Einfluss auf die strategische Planung der Entwicklungszusammenarbeit und keine Kontrolle über entwicklungspolitisch relevante Budgets.

Ein gegenläufiges zweites Szenario wäre die Bildung eines starken EAD, der für die gesamte Programmplanung und Überwachung der europäischen EZ sowie die Kontrolle über deren Finanzierungsinstrumente jenseits regionaler Zuständigkeiten verantwortlich wäre. Eine solche Lösung favorisieren die großen Mitgliedsstaaten der EU - allen voran Frankreich - aber auch Akteursgruppen, die Entwicklungspolitik im Sinne eines erweiterten Sicherheitsbegriffes als Teil der Außen- und Sicherheitspolitik begreifen. 
Eine Kompromissversion, die Elemente aus beiden Szenarien enthält, wäre zum einen die Etablierung einer gestärkten Generaldirektion Entwicklung durch die Zusammenfassung aller bislang regional aufgeteilten entwicklungspolitischen Zuständigkeiten. Um die Kohärenz innerhalb der Außenbeziehungen der EU zu gewährleisten, sieht ein Kompromissvorschlag jedoch zum anderen die Einbindung des EAD in die Programmierungsabläufe der Entwicklungszusammenarbeit vor. Vor diesem Hintergrund lässt sich nun abschließend der Vorschlag Ashtons darstellen.

\section{Der Vorschlag zum Aufbau eines Europäischen Auswärtigen Dienstes}

Insgesamt umfasst Ashtons Vorschlag 12 Artikel. Er beschäftigt sich neben der rechtlichen Natur und dem Arbeitsbereich des EADs auch mit seinen Aufgaben, der Zusammenarbeit mit anderen Organen der EU, seiner Verwaltungsstruktur und personellen Zusammensetzung. Außerdem widmet sich der Vorschlag der Frage nach dem künftigen Budget des EAD und seinen Kompetenzen innerhalb verschiedener Politikbereiche der europäischen Außenbeziehungen. In Bezug auf die Verteilung von Zuständigkeiten sieht der Vorschlag vor, die Entwicklungszusammenarbeit nicht mehr nach regionaler Zugehörigkeit der Entwicklungsländer $\mathrm{zu}$ verteilen. Kompetenzen werden stattdessen im Hinblick auf unterschiedliche Schritte innerhalb der Programmierung verteilt.

Die Kontrolle über entwicklungsrelevante Budgets soll nach dem Vorschlag innerhalb der Kommission bleiben. So fällt künftig nicht nur der Europäische Entwicklungsfonds, sondern auch das Instrument für die Entwicklungszusammenarbeit unter den primären Verantwortungsbereich des Kommissars für Entwicklung. Allerdings erhält der EAD die Möglichkeit der Einflussnahme auf die ersten drei Programmierungsschritte für die europäische Entwicklungszusammenarbeit und zwar unabhängig von der regionalen Zugehörigkeit der betroffenen Länder. Somit kann der EAD künftig auch über Fragen der langfristigen Allokation von Geldern zumindest mitentscheiden - und übt dadurch auch eine gewisse Kontrolle aus. Konkret heißt es in Artikel 8 Absatz 3:

"The European External Action Service shall in particular have responsibility for preparing the following Commission decision on the strategic, multi-annual steps within the programming cycle: (i) country allocations to determine the global financial envelope for each region [...] (ii) country and regional strategic papers (CSPs/RSPs) [and] (iii) national and regional strategic papers (NIPs/RIPs)“.

Um dieser Aufgabe gerecht zu werden, soll innerhalb des Europäischen Auswärtigen Dienstes eine Referatsstruktur geschaffen werden, die alle Weltregionen innerhalb seiner geographischen Ressorts umfasst und somit die ursprünglich nach geographisch getrennten Aufgabenbereichen agierende Verwaltung ablöst. Die relativ starke Einbindung des EAD in die Programmierung der Entwicklungszusammenarbeit ermöglicht es dem Dienst, künftig wesentlichen Einfluss auf die strategische Ausrichtung der europäischen Entwicklungszusammenarbeit zu nehmen. Allerdings bleibt die Programmierung in Bezug auf die Vergabe von Geldern aus dem Europäischen Entwicklungsfonds und die Vergabe von Geldern 
durch das Development Cooperation Instrument unter der Verantwortung des Kommissars für Entwicklung. In dem Vorschlag heißt es dazu konkret in Artikel 8 Absatz 4:

"With regard to the European Development Fund and the Development Cooperation Instrument, any proposals [...] shall be prepared [...] under the direct supervision and guidance of the Commissoner responsible for Development Policy and then jointly submitted with the High Representative for decision by the Commission."

\section{Fazit und Ausblick}

Wie ist Ashtons Vorschlag abschließend im Hinblick auf die anfangs gestellte Frage nach dem Potential der Neuerungen für die europäische Entwicklungszusammenarbeit zu bewerten? Insgesamt handelt es sich hier um einen Kompromissvorschlag, der die Entwicklungspolitik weder vollständig der Kommission noch dem Europäischen Auswärtigen Dienst zuordnet.

Durch die Einbindung des Europäischen Auswärtigen Dienstes in die strategische Planung der Entwicklungszusammenarbeit besteht künftig das Potential, eine kohärentere Außendarstellung der EU zu erreichen. Dazu benötigt der EAD jedoch fachliche entwicklungspolitische Expertise innerhalb seiner Referate. Dies sollte bei der Besetzung von Posten entsprechend berücksichtigt werden. Die institutionellen Umstrukturierungen durch eine weitere Konzentration entwicklungspolitischer Kompetenzen innerhalb der Kommission unter dem Dach der Generaldirektion Entwicklung schaffen die Voraussetzungen für eine künftig effektivere und effizientere Entwicklungszusammenarbeit.

Die Gefahr einer Instrumentalisierung der Entwicklungspolitik durch außen- oder sicherheitspolitische Interessen besteht kaum, da dem Entwicklungskommissar eine hervorgehobene Rolle innerhalb der Planung zugesprochen wird. So verbleibt die Kontrolle über die europäische Entwicklungspolitik weiterhin großenteils bei der Kommission. Allerdings bleibt abzuwarten, wie die Führungsrolle der Kommission in der Praxis ausgestaltet werden wird. Aus entwicklungspolitischer Perspektive wäre es wünschenswert, sicherzustellen, dass Gelder aus dem europäischen Entwicklungsbudget ausschließlich zur Armutsbekämpfung verwendet werden. Der Vorschlag Ashtons ist somit aus entwicklungspolitischer Perspektive eher eine Chance als ein Risiko. Alles weitere hängt von seiner konkreten Ausgestaltung ab. 